OPEN ACCESS

Edited by: Norman Poole,

St George's University, United Kingdom

Reviewed by:

Daniel P. Cardinali,

UCA Pontificia Universidad Católica Argentina, Argentina

Trevor Ronald Norman,

The University of Melbourne, Australia

*Correspondence:

Ahmet Yardimo ayardimci@firat.edu.tr

Specialty section:

This article was submitted to

Psychopharmacology,

a section of the journal

Frontiers in Psychiatry

Received: 16 January 2021 Accepted: 07 June 2021

Published: 29 June 2021

Citation:

Yardimci A, Ozdede MR and Kelestimur H (2021) Agomelatine, A

Potential Multi-Target Treatment Alternative for Insomnia, Depression, and Osteoporosis in Postmenopausal Women: A Hypothetical Model.

Front. Psychiatry 12:654616. doi: 10.3389/fpsyt.2021.654616

\section{Agomelatine, A Potential Multi-Target Treatment Alternative for Insomnia, Depression, and Osteoporosis in Postmenopausal Women: A Hypothetical Model}

\author{
Ahmet Yardimci*, Mehmet Ridvan Ozdede and Haluk Kelestimur \\ Department of Physiology, Faculty of Medicine, Firat University, Elazig, Turkey
}

Insomnia, which is associated with menopausal depression, is a common symptom of menopause. Both symptoms have a common etiology, and can affect each other significantly. Pharmacological interventions, including hypnotics and antidepressants, and non-pharmacological therapies are generally administered in clinical practice for insomnia treatment. As another menopausal disorder, osteoporosis is described as a disease of low bone mineral density (BMD), affecting nearly 200 million women worldwide. Postmenopausal osteoporosis is common among middle-aged women. Since postmenopausal osteoporosis mainly results from low estrogen levels, menopausal hormone therapy $(\mathrm{HT})$ is considered the first-line option for the prevention of osteoporosis during the menopausal period. However, almost no study has evaluated novel treatments for the combined prevention of insomnia, depression, and osteoporosis. Hence, it is necessary to develop new multi-target strategies for the treatment of these disorders to improve the quality of life during this vulnerable period. Melatonin is the major regulator of sleep, and it has been suggested to be safe and effective for bone loss therapy by MT-2 receptor activity. As a result, we hypothesize that agomelatine, an MT-1 and MT-2 receptor agonist and $5-\mathrm{HT}_{2} \mathrm{C}$ receptor antagonist, holds promise in the combined treatment of insomnia, depression, and osteoporosis in middle-aged women during menopause.

Keywords: menopause, insomnia, depression, osteoporosis, melatonin, agomelatine

\section{INTRODUCTION}

Menopause, described as the termination of menstruation, indicates a significant transition regarding reproductive status in women. The menopausal transition (MET), starting 4-6 years before the discontinuation of menses (1), is associated with hormonal fluctuations and physiological and psychological symptoms, such as sleep disturbances, hot flashes (HF), and mood changes-the frequency, severity, and duration of which may vary among women (2). Among these symptoms, as a climacteric vasomotor symptom (VMS) (3), HF is a criterion for MET and has been reported to occur in about $80 \%$ of women (1). Hot flashes includes a sensation of heat, sweating, and chills (4). Globally, about 470 million women are in the postmenopausal phase; the number of which increases by 1.5 million each year, and it is expected to reach 1.2 billion by 2030 . 
Approximately $50-85 \%$ of these women will experience VMS associated with menopause (5). In several studies, the relationship between sleep disorders and VMS has been investigated. Findings of a previous study, conducted among 962 midlife women, revealed a marked relationship between sleep disorders and VMS. About $81.3 \%$ of these women had severe VMS and poor-quality sleep; and $43.8 \%$ of them met the criteria for chronic insomnia (6). Additionally, the severity of insomnia was reported to have a correlation with HF and night sweats, and these symptoms are associated with a large-scale possibility of a diagnosis of insomnia (28.5\% in women with HF and $10.5 \%$ in women without HF) (6). In accordance with the "domino effect theory," HF disrupts sleep and leads to insomnia, which then increases vulnerability to depression. As a result, insomnia follows sleep disruption, and depression follows insomnia in a vicious cycle $(6,7)$. Additionally, Caruso et al. (2) reported that age-related irregularities in circadian rhythm may have a role as a mechanism preceding both sleep and mood disorders.

\section{BIDIRECTIONAL RELATIONSHIP BETWEEN INSOMNIA AND MAJOR DEPRESSION IN MENOPAUSE}

Clinical depression (including major depression) is known to be widespread with lifelong rates for women being 1.52 times higher than those for men. It is a complicated disorder, having several factors relevant to emotional, physical, and functional morbidity (8). Women may be at increased risk of depression during hormonal periods, such as MET, puberty, and pregnancy (9); and depressive symptoms are known to worsen throughout MET (1). Insomnia is described as a difficulty in initiating or sustaining sleep or the feeling that sleep is non-restorative, despite adequate opportunity for sleep (10). Insomnia is suggested to have a close relationship with depression and depressive disorders along the MET period (4). Insomnia symptoms are one of the most common complaints reported during the peri- and post-menopausal periods (11). Approximately $25-30 \%$ of the population have symptoms of insomnia, and this rate increases to $39-60 \%$ among women in the peri-menopausal period. Insomnia has been shown to increase the risk of developing depressive symptoms by 2-3 times during the peri-menopausal period of a woman's life (2), and more importantly, it often triggers depression (11). A 13year mental health longitudinal study by the Study of Women's Health Across the Nation (SWAN) revealed that insomnia may have a contribution to the permanence and relapse of major depressive disorder (MDD) throughout menopause (8). Hence, it seems that women having sleep problems at MET may be at risk of depression in subsequent years. Disturbed sleep, a major criterion of depression according to the Diagnostic and Statistical Manual of Mental Disorders, Fifth Edition (DSM5 ), is prevalent among women having clinical depression (8). Considering this relationship between insomnia and depression, women with menopausal insomnia seem likely to be at greater risk of depression due to the additional burden of poor sleep. Thus, the determination of safe and effective treatments for menopause-related insomnia disorders that also ameliorate comorbid depressive symptoms is advisable (11). All these results indicate that alleviating insomnia would improve not only insomnia symptoms but also depressive symptoms. Tal et al. (5) reported that treatment for sleep disturbance enhanced treatment of depression or alleviated depressive symptoms altogether.

\section{INSOMNIA TREATMENT BY NON-HORMONAL PHARMACOLOGICAL OPTIONS}

For the diagnosis and treatment of insomnia, cognitive behavioral therapy for insomnia (CBT-I) is proposed as the firstline treatment for chronic insomnia in adults of any age in the general population. However, pharmacological alternatives are recommended when CBT-I is not effective or available (7). These options include benzodiazepines, non-benzodiazepine hypnotics (Z-drugs), antidepressants, antipsychotics, and melatonin as non-hormonal pharmacological options (12). Additionally, menopausal hormone therapy (HT) is effective for sleep disorders in menopausal women (7). However, results based on several clinical studies investigating the effects of HT on insomnia in menopause are inconsistent (13), and numerous factors are involved in this treatment process, including the range of treatment protocols, dosages, and formulations (14). More importantly, a recent meta-analysis involving a search of 424 defined articles, from which 42 trials, showed that only menopausal women with VMS have an improvement in the quality of life with HT (15). Therefore, non-hormonal interventions, including antidepressants, seem likely to be better for use in this context for treating insomnia in this patient population. The use of antidepressants is not recommended in the treatment of menopause-related insomnia unless depression is present (12). Given that insomnia and depression have high comorbidity $(16,17)$ and shared etiology $(18,19)$, gold standard treatments for insomnia relevant to menopause should also ideally treat comorbid depressive symptoms in peri- and postmenopausal women at risk for depression (11). As mentioned before, since insomnia is the main criterion for depression according to DSM-5, insomnia treatment in menopausal women is also of great importance for the treatment of depression. Additionally, it would provide an alternative strategy for the indirect treatment of osteoporosis, which has a direct relationship with depression during menopause.

\section{THE EFFECT OF DEPRESSION ON MENOPAUSE-INDUCED OSTEOPOROSIS}

Osteoporosis is defined as a disease of low bone mineral density (BMD) or excessive bone loss, affecting 200 million women worldwide (20). Postmenopausal osteoporosis is prevalent in middle-aged women. Half the population of postmenopausal women have been suggested to suffer from an osteoporotic fracture in their lifetime, and those who have had a fracture are at high risk of subsequent fractures (21). More importantly, fractures resulting from osteoporosis are known to be more widespread than stroke, myocardial infarction, and breast cancer, combined. For women aged 50 years, the risk of a 
fracture due to osteoporosis is $50 \%$ in their lifetime (22). As mentioned before, depression is a disease that affects women more than men (8) and is directly related to decreased BMD (20). The relationship between menopause-induced depression and osteoporosis reveals that depression is a risk factor for osteoporosis during the postmenopausal period. During this period, women with depression have lower BMD, and are more likely to have osteoporosis than non-depressed postmenopausal women (20).

\section{AGOMELATINE}

Agomelatine is an antidepressant that combines nonmonoaminergic signaling with the classical monoaminergic mechanism. It was first introduced to the market in 2009 and was licensed for MDD treatment in adults (23). It acts as an agonist of melatonergic-1 (MT-1) and melatonergic-2 (MT-2) receptors, and as a neutral antagonist for serotonergic $\left(5-\mathrm{HT}_{2 \mathrm{C}}\right)$ receptors (24). Agomelatine is beneficial in MDD treatment and effective in the alleviation of sleep complaints (25). Agomelatine's monoaminergic and melatonergic mechanisms of action restore sleep and increase mood throughout the day. As a result, patients' quality of life is improved (25). Melatonergic and $5-\mathrm{HT}_{2 \mathrm{C}}$ receptors are expressed in the suprachiasmatic nucleus, cerebral cortex, hippocampus, amygdala, and thalamus, which are involved in the pathophysiology of depression (26). MT-1 receptor expression has a daily rhythm regulated by daylight and the internal clock. Likewise, the expression of $5-\mathrm{HT}_{2 \mathrm{c}}$ receptor mRNA has a circadian rhythm. Hence, agomelatine can be said to have a two-way effect. At night, the melatonin system dominates and favors sleep, whereas diurnal dominant $5-\mathrm{HT}_{2 \mathrm{c}}$ blockade favors wakefulness (27). Interactivity between $5-\mathrm{HT}_{2 c}$ and MT receptors contributes to agomelatine's efficacy in depression by resynchronizing disrupted circadian rhythms (28). The binding affinities, half-life, and relative potencies of agomelatine and melatonin are presented in Table $\mathbf{1}$ (29).

\section{AGOMELATINE AND MELATONIN IN DEPRESSION, INSOMNIA, AND BONE ACTIVITY}

A recent systematic review involved randomized double-blind controlled clinical trials on patients with mood disorders, including MDD. In these studies reviewed, melatonin was

TABLE 1 | Some properties of melatonin and agomelatine (29).

\begin{tabular}{lcc}
\hline & Melatonin & Agomelatine \\
\hline Binding affinity & $\mathrm{MT}_{1}: 0.085 \mathrm{nM}$ & $\mathrm{MT}_{1}: 0.062 \mathrm{nM}$ \\
& $\mathrm{MT}_{2}: 0.263 \mathrm{nM}$ & $\mathrm{MT}_{2}: 0.268 \mathrm{nM}$ \\
Half-life & $45 \mathrm{~min}$ & $1-2 \mathrm{~h}$ \\
Protein binding & $70 \%$ & $95 \%$ \\
Relative potency & $\mathrm{MT}_{1}: 1$ & $\mathrm{MT}_{1}: 1$ \\
& $\mathrm{MT}_{2}: 1$ & $\mathrm{MT}_{2}: 1$
\end{tabular}

used as an augmentation strategy in MDD (30). Among these studies, Dolberg et al. (31) revealed that using 5-10 mg slow-release (SR) melatonin with $20 \mathrm{mg}$ of fluoxetine had no effect on MDD. Similarly, Serfaty et al. (32), using $6 \mathrm{mg}$ melatonin only, had no effect. A third study, however, revealed that buspirone $(15 \mathrm{mg})$ combined with melatonin-SR ( $3 \mathrm{mg}$ ) elicited a more significant antidepressant effect than placebo or buspirone monotherapy (33). Consistent with these results, a systematic review and meta-analysis showed that melatonin had no therapeutic or prophylactic effect against depression or depressive symptoms (34). Therefore, melatonin may be thought to not have an antidepressant effect per se (35). Consequently, the antidepressant activity of melatonin has been suggested as controversial (35). According to expert opinion, the evidence supporting the clinical use of melatonin should be carefully reviewed (30). Regarding agomelatine, most reviews consensually support the idea that agomelatine has antidepressant efficacy (36-40). Moreover, according to a meta-analysis study, the antidepressant efficacy of agomelatine has been established by 20 trials with 7,460 participants that agomelatine was significantly more effective than placebo in MDD (41). Doses of 25-50 $\mathrm{mg} /$ day have been used in comparative experiments and placebocontrolled trials. The doses of agomelatine have been indicated to be within this range (42). However, almost no study has investigated the effects of agomelatine on MDD in menopausal women. Heun et al. (43) in comparing the effectiveness of agomelatine (25-50 mg/day p.o.) to that of placebo in an 8week treatment schedule of elderly patients ( $\geq 65$ years old) with recurrent $\mathrm{MDD}$, proposed agomelatine (25-50 $\mathrm{mg} /$ day) to be more effective and well tolerated in elderly patients with depression. Additionally, agomelatine has been shown to be well tolerated by patients with only minimum deviations from placebo, and it has a good safety profile in such patients, including elderly patients (43). Besides, $69.5 \%$ of the 151 patients who received agomelatine were elderly women in this study. To the best of our knowledge, however, no study compared the antidepressant activity of agomelatine and melatonin on menopausal period in the literature. Thus, apart from melatonin, the current results suggest that agomelatine may be addressed as a reasonable choice for the elderly population, including menopausal women because of its good tolerability, effectiveness on symptoms, and better adverse effect profile.

As mentioned earlier, there is a bidirectional relationship between insomnia and MDD in menopause. Insomnia has been suggested to increase the risk of experiencing depressive symptoms by 2-3 times during the peri-menopausal period (2), and it often causes depression (11). Melatonin has been suggested to relieve all subjective sleep symptoms of postmenopausal women with insomnia (7). Additionally, melatonin has a moderate hypnotic effect in insomnia treatment and does not cause morning hangover symptoms (44-46). In the last few decades, prolonged-release melatonin (PRM) (2 mg) for insomnia with reduced sleep quality in people over 55 years old has been accepted $(47,48)$. It is the only melatonin-containing drug that was accepted, and its prolonged-release mechanism mimics the internal melatonin secretion pattern by progressively releasing melatonin (7). PRM (2 mg) improved sleep efficiency, 
morning wakefulness, and quality of life, as well as sleep latency, among people aged 55-80 years-these benefits were sustained or augmented over 6 months (47). The majority of hypnoticrelated safety issues do not arise with PRM (2 mg). Accordingly, PRM (2 mg) can be suggested as a useful therapeutic option for menopausal women and stands for a safer choice over benzodiazepine or Z-drugs (7).

Regarding the effects of agomelatine on insomnia, it is suggested to have benefits with initial insomnia. Additionally, it increases sleep duration and performance, and decreases daytime drowsiness. However, it does not affect the sleep architecture of patients with depression (49). In a randomized double-blind controlled study, agomelatine presented significantly better improvement than venlafaxine on insomnia in patients with depression (28). In another randomized double-blinded study, Quera-Salva et al. (50) showed that agomelatine more significantly reduced sleep latency than escitalopram. In randomized clinical trials comparing agomelatine to selective serotonin reuptake inhibitors (SSRIs) and venlafaxine, agomelatine enhanced all parts of the sleep-wake cycle, especially during the initial stages of sleep and sleep quality, as well as daytime alertness (51). Agomelatine has also been found to be effective in alleviating circadian rhythm disturbances reported in patients with MDD (29). Melatonin treatment in menopause for insomnia has been suggested to reduce sleep disturbances during menopause. Although no study directly compares melatonin and agomelatine in the treatment of insomnia, agomelatine seems to hold promise for insomnia treatment in menopausal women.

Melatonin is known to have positive effects on bone health and is suggested to have likely therapeutic potential on postmenopausal osteoporosis (52). Li et al. (53) reviewed the role and importance of melatonin in bone metabolism. In relation to the effects of melatonin on osteoporosis, Sharan et al. (52) showed that melatonin reverses bone loss by greatly increasing bone formation in ovariectomized mice, which are the animal models of menopause-induced osteoporosis. According to the study findings, MT-2 is the receptor responsible for the effect of melatonin on osteoblasts, whereas inactivation of MT-1 does not affect bone mass. Conversely, agomelatine, as an MT-1 and MT-2 agonist, has not been studied in osteoporosis occurring among menopausal women. However, one recent study showed that agomelatine has ameliorative effects on bone fracture healing in rodents (54). Bone mass is known to be formed by either excessive osteoclastic bone resorption or reduced osteoblastic bone formation. The bone remodeling process starts by proliferation, differentiation, and activation of mononuclear precursors. Two crucial proteins have important roles in these processes: receptor activator of NF-кB ligand (RANKL) and its soluble decoy receptor osteoprotegerin (OPG). RANKL binds to its cellular receptor RANK and stimulates differentiation and activation in osteoclasts resorbing bone. Osteoprotegerin inhibits osteoclastogenesis by binding and inactivating RANKL (55). Zhou et al. (56) reported that RANKL was suppressed by melatonin. Compatible with these findings, in the same study, the OPG/RANKL ratio, which is an important determinant of bone mass and skeletal integrity (57), has been suggested to be elevated by melatonin in bone marrow mesenchymal stem cells. Additionally, NF- $\kappa \mathrm{B}$ was inactivated via the MT-2 receptor, which is responsible for melatonin-regulated osteogenesis. Hence, considering all these results, agomelatine may possibly have such an effect via the MT-2 receptor on osteoporosis in menopausal women.

\section{THE HYPOTHESIS}

Guided by literature, we hypothesize that agomelatine may be a new alternative in the combined treatment of insomnia, depression, and osteoporosis in menopausal women, treating these three disorders concurrently in comorbid conditions.

\section{EVALUATION OF THE HYPOTHESIS}

Insomnia and depression are known to have a mutual relationship without clarity of the direction of effect on each other (58). This relationship also indicates that the association between depression and insomnia is not simply a cause-effect relationship, but instead a complex bidirectional one (59). Altogether, treatment of insomnia most likely may positively affect depressive symptoms in menopausal women due to their comorbidity. In a recent study supporting this situation, insomnia treatment was reported to significantly contribute to the reduction of depressive symptoms (11). Hypnotics and antidepressants are generally prescribed for the treatment of patients with comorbid of depression and sleep problems. However, hypnotics may lead to depression (59). Benzodiazepine hypnotics have many different side effects, including next-day hangover and rebound insomnia, and the next-day hangover is reported to be the most frequent complaint reported by patients using benzodiazepines. Benzodiazepines were suggested to significantly decrease sleep latency and increased total sleep duration (60). However, they are associated with significant adverse events on long-term use (60). Concerning SSRIs as a treatment for insomnia, a comprehensive review of the literature suggests that escitalopram is a safer alternative, as an SSRI, among the various antidepressants, especially with comorbid depression, for the treatment of insomnia in menopause (13).

However, SSRIs are suggested to probably increase fracture risk by decreasing BMD. Conversely, according to a US randomized placebo-controlled trial conducted that assessed the effect of escitalopram on bone turnover markers, escitalopram does not change it in short-term usage. Additionally, the trial confirmed that the result of the study could not be generalized for the long-term use of other SSRIs (61). Moreover, antidepressants in this group have many different side effects (62), and one of the most prominent of these side effects was reported to be on sleep (60). From this point of view, since agomelatine has a better side-effect profile, efficacy, and tolerability in the treatment of depression than SSRIs, as an antidepressant and sleep regulator, due to its ability to alleviate complaints about sleep disorders (60), it stands out as a new multi-target 
alternative for insomnia, depression, and osteoporosis treatment with minimum adverse effects.

\section{CONCLUSION}

Regarding the domino effect observed in the vicious cycle involving insomnia and depression, the use of agomelatine, an antidepressant that has been proven effective in both disorders, to break this cycle would reduce both the treatment burden and global financial burden of drug consumption for patients with comorbidity insomnia and depression. Concurrently, the treatment is predicted to contribute to the improvement of osteoporosis, which seriously affects the quality of life of middle-aged women and causes bone tissue fractures in subsequent years. This bidirectional therapeutic effect provided by agomelatine is very important, since improvements in sleep in patients with depression are associated with a reduction in the recurrence rate of depressive symptoms (60). Therefore, the ameliorative effect to be achieved with agomelatine in patients with comorbid depression and insomnia would ensure that both disorders would be cured along with osteoporosis.

\section{FUTURE PERSPECTIVE}

If our hypothesis is correct, agomelatine would come into prominence as a multi-target agent due to its curative effect on insomnia, depression, and osteoporosis, which are commonly seen in menopausal women. However, some important points need to be stressed. First, the use of agomelatine would seem

\section{REFERENCES}

1. Baker FC, De Zambotti M, Colrain IM, Bei B. Sleep problems during the menopausal transition: prevalence, impact, and management challenges. Nat Sci Sleep. (2018) 10:73-95. doi: 10.2147/NSS.S125807

2. Caruso D, Masci I, Cipollone G, Palagini L. Insomnia and depressive symptoms during the menopausal transition: theoretical and therapeutic implications of a self-reinforcing feedback loop. Maturitas. (2019) 123:78-81. doi: 10.1016/j.maturitas.2019.02.007

3. Ohayon MM. Severe hot flashes are associated with chronic insomnia. Arch Intern Med. (2006) 166:1262-8. doi: 10.1001/archinte.166.12.1262

4. Composto J, Leichman ES, Luedtke K, Mindell JA. Thermal comfort intervention for hot-flash related insomnia symptoms in perimenopausal and postmenopausal-aged women: an exploratory study. Behav Sleep Med. (2021) 19:38-47. doi: 10.1080/15402002.2019.1699100

5. Tal JZ, Suh SA, Dowdle CL, Nowakowski S. Treatment of insomnia, insomnia symptoms, and obstructive sleep apnea during and after menopause: therapeutic approaches. Curr Psychiatry Rev. (2015) 11:63-83. doi: 10.2174/1573400510666140929194848

6. Bonanni E, Schirru A, Di Perri MC, Bonuccelli U, Maestri M. Insomnia and hot flashes. Maturitas. (2019) 126:51-4. doi: 10.1016/j.maturitas.2019.05.001

7. Proserpio P, Marra S, Campana C, Agostoni EC, Palagini L, Nobili L, et al. Insomnia and menopause: a narrative review on mechanisms and treatments. Climacteric. (2020) 23:539-49. doi: 10.1080/13697137.2020.1799973

8. Bromberger JT, Kravitz HM, Youk A, Schott LL, Joffe H. Patterns of depressive disorders across 13 years and their determinants among midlife women: SWAN mental health study. J Affect Disord. (2016) 206:31-40. doi: 10.1016/j.jad.2016.07.005

9. Vivian-Taylor J, Hickey M. Menopause and depression: is there a link? Maturitas. (2014) 79:142-6. doi: 10.1016/j.maturitas.2014.05.014 to be a rational choice for middle-aged women comorbid with depression and insomnia due to its bidirectional effects. Additionally, since agomelatine has a good therapeutic potential for depression, it may have an indirect preventive role against osteoporosis, given that depression itself is an important risk factor for osteoporosis. Based on a recent study, it can as well directly heal osteoporosis through MT-2 receptors (52). Concerning agomelatine's antidepressant and sleep constructive effect in menopausal women, only one pilot study has been conducted to date (63). Seemingly, agomelatine, as a novel therapeutic avenue for being a multi-target drug, would treat all three diseases concurrently in this specific patient population, since health issues (especially for insomnia and depression) in middle-aged women are likely linked together and coexist.

\section{DATA AVAILABILITY STATEMENT}

The original contributions presented in the study are included in the article/supplementary material, further inquiries can be directed to the corresponding author/s.

\section{AUTHOR CONTRIBUTIONS}

AY developed the hypothesis and wrote the manuscript. MRO contributed to the literature review and manuscript editing. HK is the coordinator of the paper, he critically revised the article for important intellectual content. All authors contributed to the article and approved the submitted version.

10. Bruyneel M. Sleep disturbances in menopausal women: aetiology and practical aspects. Maturitas. (2015) 81:406-9. doi: 10.1016/j.maturitas.2015.04.017

11. Kalmbach DA, Cheng P, Arnedt JT, Anderson JR, Roth T, Fellman-Couture $\mathrm{C}$, et al. Treating insomnia improves depression, maladaptive thinking, and hyperarousal in postmenopausal women: comparing cognitive-behavioral therapy for insomnia (CBTI), sleep restriction therapy, and sleep hygiene education. Sleep Med. (2019) 55:124-34. doi: 10.1016/j.sleep.2018.11.019

12. Caretto M, Giannini A, Simoncini T. An integrated approach to diagnosing and managing sleep disorders in menopausal women. Maturitas. (2019) 128:1-3. doi: 10.1016/j.maturitas.2019.06.008

13. Attarian H, Hachul H, Guttuso T, Phillips B. Treatment of chronic insomnia disorder in menopause: evaluation of literature. Menopause. (2015) 22:67484. doi: 10.1097/GME.0000000000000348

14. Hachul H, Polesel DN. Insomnia pharmacotherapy: a review of current treatment options for insomnia in menopause. Curr Sleep Med Rep. (2017) 3:299-305. doi: 10.1007/s40675-017-0090-3

15. Cintron D, Lipford M, Larrea-Mantilla L, Spencer-Bonilla G, Lloyd R, Gionfriddo MR, et al. Efficacy of menopausal hormone therapy on sleep quality: systematic review and meta-analysis. Endocrine. (2017) 55:702-11. doi: 10.1007/s12020-016-1072-9

16. Ohayon MM, Roth T. Place of chronic insomnia in the course of depressive and anxiety disorders. J Psychiatr Res. (2003) 37:9-15. doi: 10.1016/s0022-3956(02)00052-3

17. Kalmbach DA, Pillai V, Arnedt JT, Drake CL. DSM-5 insomnia and short sleep: comorbidity landscape and racial disparities. Sleep. (2016) 39:2101-11. doi: $10.5665 /$ sleep.6306

18. Fernández-Mendoza J, Vela-Bueno A, Vgontzas AN, Ramos-Platón MJ, Olavarrieta-Bernardino S, Bixler EO, et al. Cognitive-emotional hyperarousal as a premorbid characteristic of individuals vulnerable to insomnia. Psychosom Med. (2010) 72:397-403. doi: 10.1097/PSY.0b013e3181d75319 
19. Hammerschlag AR, Stringer S, de Leeuw CA, Sniekers S, Taskesen E, Watanabe K, et al. Genome-wide association analysis of insomnia complaints identifies risk genes and genetic overlap with psychiatric and metabolic traits. Nat Genet. (2017) 49:1584-92. doi: 10.1038/ng.3888

20. Mollard E, Bilek L, Waltman N. Emerging evidence on the link between depressive symptoms and bone loss in postmenopausal women. Int J Womens Health. (2017) 10:1-9. doi: 10.2147/IJWH.S147006

21. Eastell R, Rosen CJ, Black DM, Cheung AM, Murad MH, Shoback D. Pharmacological management of osteoporosis in postmenopausal women: an Endocrine Society clinical practice guideline. J Clin Endocrinol Metab. (2019) 104:1595-622. doi: 10.1210/jc.2019-00221

22. Watts NB. Postmenopausal osteoporosis: a clinical review. J Womens Health. (2018) 27:1093-6. doi: 10.1089/jwh.2017.6706

23. Konstantakopoulos G, Dimitrakopoulos S, Michalopoulou PG. The preclinical discovery and development of agomelatine for the treatment of depression. Expert Opin Drug Discov. (2020) 15:1121-32. doi: 10.1080/17460441.2020.1781087

24. Tardito D, Molteni R, Popoli M, Racagni G. Synergistic mechanisms involved in the antidepressant effects of agomelatine. Eur Neuropsychopharmacol. (2012) 22:482-6. doi: 10.1016/j.euroneuro.2012.06.016

25. Pandi-Perumal SR, Moscovitch A, Srinivasan V, Spence DW, Cardinali DP, Brown GM. Bidirectional communication between sleep and circadian rhythms and its implications for depression: lessons from agomelatine. Prog Neurobiol. (2009) 88:264-71. doi: 10.1016/j.pneurobio.2009.04.007

26. San L, Arranz B. Agomelatine: a novel mechanism of antidepressant action involving the melatonergic and the serotonergic system. Eur Psychiatry. (2008) 23:396-402. doi: 10.1016/j.eurpsy.2008.04.002

27. Cerit C, Yalug I, Akpinar E, Talas A, Tufan AE, Özten E. Agomelatin depresyon tedavisine ne getiriyor? Gün. Gözd. Geçir. Yeni Symp. (2013) 51:3.

28. Lemoine P, Guilleminault C, Alvarez E. Improvement in subjective sleep in major depressive disorder with a novel antidepressant, agomelatine: randomized, double-blind comparison with venlafaxine. J Clin Psychiatry. (2007) 68:1723-32. doi: 10.4088/jcp.v68n1112

29. Cardinali DP, Srinivasan V, Brzezinski A, Brown GM. Melatonin and its analogs in insomnia and depression. J Pineal Res. (2012) 52:365-75. doi: 10.1111/j.1600-079X.2011.00962.X

30. Tonon AC, Pilz LK, Markus RP, Hidalgo MP, Elisabetsky E. Melatonin and Depression: a translational perspective from animal models to clinical studies. Front Psychiatry. (2021) 12:638981. doi: 10.3389/fpsyt.2021.638981

31. Dolberg OT, Hirschmann S, Grunhaus L. Melatonin for the treatment of sleep disturbances in major depressive disorder. Am J Psychiatry. (1998) 155:1119-21. doi: 10.1176/ajp.155.8.1119

32. Serfaty MA, Osborne D, Buszewicz MJ, Blizard R, Raven PW. A randomized double-blind placebo-controlled trial of treatment as usual plus exogenous slow-release melatonin $(6 \mathrm{mg})$ or placebo for sleep disturbance and depressed mood. Int Clin Psychopharmacol. (2010) 25:13242. doi: 10.1097/YIC.0b013e32832c260b

33. Fava M, Targum SD, Nierenberg AA, Bleicher LS, Carter TA, Wedel PC, et al. An exploratory study of combination buspirone and melatonin SR in major depressive disorder (MDD): a possible role for neurogenesis in drug discovery. J Psychiatr Res. (2012) 46:1553-63. doi: 10.1016/j.jpsychires.2012.08.013

34. Hansen MV, Danielsen AK, Hageman I, Rosenberg J, Gögenur I. The therapeutic or prophylactic effect of exogenous melatonin against depression and depressive symptoms: a systematic review and meta-analysis. Eur Neuropsychopharmacol. (2014) 24:1719-28. doi: 10.1016/j.euroneuro.2014.08.008

35. Norman TR. “Agomelatine, melatonin and depression". In: F. López-Muñoz, V. Srinivasan, D. de Berardis, C. Álamo, T. A. Kato, editors. Melatonin, Neuroprotective Agents and Antidepressant Therapy. 1st ed. New Delhi: Springer (2016). p. 229-47. doi: 10.1007/978-81-322-2803-5

36. Howland RH. Agomelatine: a novel atypical antidepressant. J Psychosoc Nurs Ment Health Serv. (2007) 45:13-7. doi: 10.3928/02793695-20071201-04

37. Kennedy SH. Agomelatine: efficacy at each phase of antidepressant treatment. CNS Drugs. (2009) 23(Suppl 2):41-7. doi: 10.2165/11318660-000000000-00000

38. Kennedy SH, Rizvi SJ. Agomelatine in the treatment of major depressive disorder: potential for clinical effectiveness. CNS Drugs. (2010) 24:479-99. doi: 10.2165/11534420-000000000-00000
39. Singh SP, Singh V, Kar N. Efficacy of agomelatine in major depressive disorder: meta-analysis and appraisal. Int J Neuropsychopharmacol. (2012) 15:417-28. doi: $10.1017 /$ S1461145711001301

40. Montgomery, Stuart A. Major depressive disorders: clinical efficacy and tolerability of agomelatine, a new melatonergic agonist. Eur Neuropsychopharmacol. (2006) 16:633-8. doi: 10.1016/S0924-977X(06)70009-8

41. Taylor D, Sparshatt A, Varma S, Olofinjana O. Antidepressant efficacy of agomelatine: meta-analysis of published and unpublished studies. BMJ. (2014) 348:g1888. doi: 10.1136/bmj.g1888. Erratum in: BMJ. (2014) 348:g2496.

42. Norman TR, Olver JS. Agomelatine for depression: expanding the horizons? Expert Opin Pharmacother. (2019) 20:647-56. doi: 10.1080/14656566.2019.1574747

43. Heun R, Ahokas A, Boyer P, Giménez-Montesinos N, Pontes-Soares F, Olivier $\mathrm{V}$, et al. The efficacy of agomelatine in elderly patients with recurrent major depressive disorder: a placebo-controlled study. J Clin Psychiatry. (2013) 74:587-94. doi: 10.4088/JCP.12m08250

44. Bellipanni G, Bianchi P, Pierpaoli W, Bulian D, Ilyia E. Effects of melatonin in perimenopausal and menopausal women: a randomized and placebo controlled study. Exp Gerontol. (2001) 36:297-310. doi: 10.1016/s0531-5565(00)00217-5

45. Bellipanni G, Di Marzo F, Blasi F, Di Marzo A. Effects of melatonin in perimenopausal and menopausal women: our personal experience. Ann N Y Acad Sci. (2005) 1057:393-402. doi: 10.1196/annals.1356.030

46. Gursoy AY, Kiseli M, Caglar GS. Melatonin in aging women. Climacteric. (2015) 18:790-6. doi: 10.3109/13697137.2015.1052393

47. Wade AG, Crawford G, Ford I, McConnachie A, Nir T, Laudon M, et al Prolonged release melatonin in the treatment of primary insomnia: evaluation of the age cut-off for short- and long-term response. Curr Med Res Opin. (2011) 27:87-98. doi: 10.1185/03007995.2010.537317

48. Wilson SJ, Nutt DJ, Alford C, Argyropoulos SV, Baldwin DS, Bateson $\mathrm{AN}$, et al. British Association for Psychopharmacology consensus statement on evidence-based treatment of insomnia, parasomnias and circadian rhythm disorders. J Psychopharmacol. (2010) 24:1577-601. doi: 10.1177/0269881110379307

49. De Berardis D, Marini S, Fornaro M, Srinivasan V, Iasevoli F, Tomasetti $\mathrm{C}$, et al. The melatonergic system in mood and anxiety disorders and the role of agomelatine: implications for clinical practice. Int J Mol Sci. (2013) 14:12458-83. doi: 10.3390/ijms140612458

50. Quera-Salva MA, Hajak G, Philip P, Montplaisir J, Keufer-Le Gall S, Laredo J, et al. Comparison of agomelatine and escitalopram on nighttime sleep and daytime condition and efficacy in major depressive disorder patients. Int Clin Psychopharmacol. (2011) 26:252-62. doi: 10.1097/YIC.0b013e328349b117

51. Quera-Salva MA, Lemoine P, Guilleminault C. Impact of the novel antidepressant agomelatine on disturbed sleep-wake cycles in depressed patients. Hum Psychopharmacol. (2010) 25:222-9. doi: 10.1002/hup.1112

52. Sharan K, Lewis K, Furukawa T, Yadav VK. Regulation of bone mass through pineal-derived melatonin-MT 2 receptor pathway. J Pineal Res. (2017) 63:e12423. doi: 10.1111/jpi.12423

53. Li T, Jiang S, Lu C, Yang W, Yang Z, Hu W. et al. Melatonin: another avenue for treating osteoporosis? J Pineal Res. (2019) 66:e12548. doi: 10.1111/jpi.12548

54. Köse D, Köse A, Halici Z, Gürbüz MA, Aydin A, Ugan RA, et al. Do peripheral melatonin agonists improve bone fracture healing? The effects of agomelatine and ramelteon on experimental bone fracture. Eur J Pharmacol. (2020) 887:173577. doi: 10.1016/j.ejphar.2020.173577

55. Jabbar S, Drury J, Fordham JN, Datta HK, Francis RM, Tuck SP. Osteoprotegerin, RANKL and bone turnover in postmenopausal osteoporosis. J Clin Pathol. (2011) 64:354-7. doi: 10.1136/jcp.2010.086595

56. Zhou Y, Wang C, Si J, Wang B, Zhang D, Ding D, et al. Melatonin up-regulates bone marrow mesenchymal stem cells osteogenic action but suppresses their mediated osteoclastogenesis via $\mathrm{MT}_{2}$-inactivated $\mathrm{NF}-\kappa \mathrm{B}$ pathway. $\mathrm{Br}$ J Pharmacol. (2020) 177:2106-22. doi: 10.1111/bph.14972

57. Boyce BF, Xing L. Biology of RANK, RANKL, and osteoprotegerin. Arthritis Res Ther. (2007) 9(Suppl 1):S1. doi: 10.1186/ar2165

58. Eichling PS, Sahni J. Menopause related sleep disorders. J Clin Sleep Med (2005) 1:291-300. doi: 10.5664/jcsm.26347

59. Fang $\mathrm{H}, \mathrm{Tu} \mathrm{S}$, Sheng J, Shao A. Depression in sleep disturbance: a review on a bidirectional relationship, mechanisms and 
treatment. J Cell Mol Med. (2019) 23:2324-32. doi: $10.1111 / \mathrm{jcm}$ m. 14170

60. Srinivasan V, Brzezinski A, Pandi-Perumal SR, Spence DW, Cardinali DP, Brown GM. Melatonin agonists in primary insomnia and depression-associated insomnia: are they superior to sedative-hypnotics? Prog Neuropsychopharmacol Biol Psychiatry. (2011) 1;35:913-23. doi: 10.1016/j.pnpbp.2011.03.013

61. Wadhwa R, Kumar M, Talegaonkar S, Vohora D. Serotonin reuptake inhibitors and bone health: a review of clinical studies and plausible mechanisms. Osteoporos Sarcopenia. (2017) 3:75-81. doi: 10.1016/j.afos.2017.05.002

62. Carrasco JL, Sandner C. Clinical effects of pharmacological variations in selective serotonin reuptake inhibitors: an overview. Int $J$ Clin Pract. (2005) 59:1428-34. doi: 10.1111/j.1368-5031.2005.0 0681.x
63. Krüger $S$, Tran T. EPA-1061-Agomelatine in the treatment of perimenopausal depression-a pilot study. Eur Psychiatry. (2014) 29:1-1. doi: $10.1016 / \mathrm{S} 0924-9338(14) 78345-2$

Conflict of Interest: The authors declare that the research was conducted in the absence of any commercial or financial relationships that could be construed as a potential conflict of interest.

Copyright $\odot 2021$ Yardimci, Ozdede and Kelestimur. This is an open-access article distributed under the terms of the Creative Commons Attribution License (CC BY). The use, distribution or reproduction in other forums is permitted, provided the original author(s) and the copyright owner(s) are credited and that the original publication in this journal is cited, in accordance with accepted academic practice. No use, distribution or reproduction is permitted which does not comply with these terms. 